\title{
Comparison Between Zagreb Eccentricity Indices and the Eccentric Connectivity Index, the Second Geometric-arithmetic Index and the Graovac-Ghorbani Index
}

Kinkar Ch. Das

Department of Mathematics, Sungkyunkwan University, Suwon 440-746, Republic of Korea

Author's e-mail address: kinkardas2003@gmail.com

RECEIVED: October 2, 2016 * REVISED: March 31, 2017 * ACCEPTED: April 5, 2017

THIS PAPER IS DEDICATED TO PROFESSOR NENAD TRINAJSTIĆ ON THE OCCASION OF HIS 80 ${ }^{\text {TH }}$ BIRTHDAY

\begin{abstract}
The concept of Zagreb eccentricity indices $\left(E_{1}\right.$ and $\left.E_{2}\right)$ was introduced in the chemical graph theory very recently. The eccentric connectivity index $\left(\xi^{c}\right)$ is a distance-based molecular structure descriptor that was used for mathematical modeling of biological activities of diverse nature. The second geometric-arithmetic index $\left(G A_{2}\right)$ was introduced in 2010, is found to be useful tool in QSPR and QSAR studies. In 2010 Graovac and Ghorbani introduced a distance-based analog of the atom-bond connectivity index, the Graovac-Ghorbani index $\left(A B C_{G G}\right)$, which yielded promising results when compared to analogous descriptors. In this note we prove that $E_{1}(T)>\xi^{c}(T)$ for chemical trees $T$. For connected graph $G$ of order $n$ with maximum degree $\Delta$, it is proved that $\xi^{c}(G)>E_{2}(G)$ if $\Delta=n-1$ and $\xi^{c}(G)<E_{2}(G)$, otherwise. Moreover, we show that $G A_{2}>A B C_{G G}$ for paths and some class of bipartite graphs.
\end{abstract}

Keywords: eccentric connectivity index, first Zagreb eccentricity index, second Zagreb eccentricity index, second geometric-arithmetic index, second atom-bond connectivity index.

\section{INTRODUCTION}

A topological index is a numerical descriptor of the molecular structure derived from the corresponding molecular graph. There are numerous topological descriptors that have found some applications in theoretical chemistry, especially in QSPR/QSAR research. ${ }^{[1,2]}$ They can be classified based on the structural properties of graphs used for their calculation. The following topological indices are well-studied by the researchers: Wiener index, ${ }^{[3]}$ Hosoya index ${ }^{[4]}$ the energy ${ }^{[5]}$ and the Randić connectivity index. ${ }^{[6]}$

Let $G=(V, E)$ denote a simple graph with $n$ vertices and $m$ edges, where $V(G)=\left\{v_{1}, v_{2}, \ldots, v_{n}\right\}$ and $|E(G)|=m$ (The cardinality of a set $S$, denoted $|S|$, is the number of elements in $S)$. The degree of a vertex $v_{i} \in V(G), d_{G}\left(v_{i}\right)$ is the number of edges incident to $v_{i}$. The maximum degree of a graph $G$ is denoted by $\Delta$, that is, $\Delta=\max \left\{d_{G}\left(v_{i}\right): v_{i} \in V(G)\right\}$. The distance between $v_{i}$ and $v_{j}$ in $V(G), d_{G}\left(v_{i}, v_{j}\right)$, is the length of a shortest $v_{i}$ to $v_{j}$ path in $G$. The eccentricity, $\varepsilon_{G}\left(v_{i}\right)$ of a vertex $v_{i} \in V(G)$ is the maximum distance between $v_{i}$ and any other vertex in $G$, that is, $\varepsilon_{G}\left(v_{j}\right)=\max \left\{d_{G}\left(v_{i}, v_{j}\right): v_{j} \in V(G)\right\}$. The diameter of $G, d$, is defined as the maximum value of the eccentricities of the vertices of $G$, that is, $d=\max \left\{d_{G}\left(v_{i}, v_{j}\right): v_{i}, v_{j} \in V(G)\right\}$.

Gutman and Trinajstić[7] derived a formula for estimating total $\pi$-electron energy of conjugated systems. Their formula contained two terms that later became known as the Zagreb indices $M_{1}$ and $M_{2}$. The first Zagreb index $M_{1}(G)$ and the second Zagreb index $M_{2}(G)$ of graph $G$ (see Refs. [2], [7-11] and the references therein) are among the oldest and most studied topological indices. They are defined as:

$$
M_{1}(G)=\sum_{v_{i} \in V(G)} d_{G}^{2}\left(v_{i}\right) \text { and } M_{2}(G)=\sum_{v_{i} v_{j} \in E(G)} d_{G}\left(v_{i}\right) \cdot d_{G}\left(v_{j}\right) \text {, }
$$

where $d_{G}\left(v_{i}\right)$ is the degree of the vertex $v_{i}$ of graph $G$. 
The invariants based on vertex eccentricities attracted some attention in chemistry. In an analogy with the first and the second Zagreb indices, Ghorbani et al. ${ }^{[12]}$ and Vukičević et al. ${ }^{[13]}$ defined the first $E_{1}$, and the second $E_{2}$, Zagreb eccentricity indices by

$$
E_{1}(G)=\sum_{v_{i} \in V(G)} \varepsilon_{G}^{2}\left(v_{i}\right)
$$

and

$$
E_{2}(G)=\sum_{v_{i} v_{j} \in E(G)} \varepsilon_{G}\left(v_{i}\right) \cdot \varepsilon_{G}\left(v_{j}\right)
$$

where $\varepsilon_{G}\left(v_{i}\right)$ is the eccentricity of the vertex $v_{i}$ in $G$. Upper and lower bounds for the Zagreb eccentricity indices of graphs have been reported in Refs. [12-15].

The eccentric connectivity index of a graph $G$, denoted by $\xi^{C}(G)$, is defined as ${ }^{[16]}$

$$
\xi^{c}(G)=\sum_{v_{i} \in V(G)} d_{G}\left(v_{i}\right) \cdot \varepsilon_{G}\left(v_{i}\right)=\sum_{v_{i} v_{j} \in E(G)}\left(\varepsilon_{G}\left(v_{i}\right)+\varepsilon_{G}\left(v_{j}\right)\right),
$$

where $d_{G}\left(v_{i}\right)$ and $\varepsilon_{G}\left(v_{i}\right)$ are the degree and the eccentricity of the vertex $v_{i}$ in $G$, respectively. The eccentric connectivity index provides good correlations with regard to both physical and biological properties. ${ }^{[17]}$ The simplicity amalgamated with high correlating ability of this index can be easily exploited in QSPR/QSAR studies. Such studies can easily provide valuable leads for the development of potential therapeutic agents. We encourage the reader to consult papers ${ }^{[18,19]}$ for the mathematical properties of the eccentric connectivity index.

Let $e$ be an edge of the graph $G$ (which may contain cycles or be acyclic), connecting the vertices $v_{i}$ and $v_{j}$. Here we define two sets $N_{i}(e \mid G)$ and $N_{j}(e \mid G)$ as follows:

$$
\begin{aligned}
& N_{i}(e \mid G)=\left\{v_{k} \in V(G) \mid d_{G}\left(v_{k}, v_{i}\right)<d_{G}\left(v_{k}, v_{j}\right)\right\}, \\
& N_{j}(e \mid G)=\left\{v_{k} \in V(G) \mid d_{G}\left(v_{k}, v_{j}\right)<d_{G}\left(v_{k}, v_{j}\right)\right\} .
\end{aligned}
$$

The number of elements of $N_{i}(e \mid G)$ and $N_{j}(e \mid G)$ are denoted by $n_{i}(e \mid G) \quad\left(=\left|N_{i}(e \mid G)\right|\right)$ and $n_{j}(e \mid G) \quad\left(=\left|N_{j}(e \mid G)\right|\right)$, respectively. Therefore $n_{i}(e \mid G)$ counts the number of vertices of $G$ lying closer to the vertex $v_{i}$ than to vertex $v_{j}$. The meaning of $n_{j}(e \mid G)$ is analogous. Vertices equidistant from both ends of the edge $v_{i} v_{j}$ belong neither to $N_{i}(e \mid G)$ nor to $N_{j}(e \mid G)$. Note that for any edge $e$ of $G$, $n_{i}(e \mid G) \geq 1$ and $n_{j}(e \mid G) \geq 1$, because $v_{i} \in N_{i}(e \mid G)$ and $v_{j} \in N_{j}(e \mid G)$. For the sake of brevity, if there is no risk of confusion, we always simplify $n_{i}(e \mid G)$ and $n_{j}(e \mid G)$ as $n_{i}$ and $n_{j}$, respectively.

Recently, Fath-Tabar, Furtula and Gutman ${ }^{[20]}$ defined second geometric-arithmetic index by

$$
G A_{2}(G)=\sum_{v_{i} v_{j} \in E(G)} \frac{\sqrt{n_{i} \cdot n_{j}}}{\frac{1}{2}\left[n_{i}+n_{j}\right]} .
$$

For the mathematical properties of $G A_{2}$ index, the reader is referred to Refs. [20-22]

In Ref. [23], Graovac and Ghorbani proposed the following distance-based analog of the $A B C$ index:

$$
A B C_{G G}(G)=\sum_{v_{i} v_{j} \in E(G)} \sqrt{\frac{n_{i}+n_{j}-2}{n_{i} \cdot n_{j}}} .
$$

Some initial studies indicate that the Graovac-Ghorbani index could be an effective predictive tool in chemistry. For instance, it can be used to model both the boiling and the melting points of molecules. ${ }^{[24]}$ Upper and lower bounds for the $A B C_{G G}$ index of graphs have been given in Refs. [23], [25-28].

Let $\sum$ be the class of finite graphs. A topological index is a function Top from $\sum$ into real numbers, where for $G$ and $H$ being isomorphic: $\operatorname{Top}(G)=\operatorname{Top}(H)$. Suppose two topological indices $T o p_{1}$ and $T o p_{2}$. Since $T o p_{1}$ and $\mathrm{Top}_{2}$ are real numbers for any graph $G$, then it is interesting to compare these two topological indices $T o p_{1}$ and $T o p_{2}$ for $G$, that is, $\operatorname{Top}_{1}(G) \geq \operatorname{Top}_{2}(G)$ or $\operatorname{Top}_{1}(G)<\operatorname{Top}_{2}(G)$ ? Recently, Das and Trinajstić compared the first geometricarithmetic index and the atom-bond connectivity index for trees and graphs. Moreover, they compared $\xi^{c}$ with $M_{1}$ and $M_{2}$ for chemical trees, molecular graphs and some graph families. Several relations between the two $A B C$-indices are established. Geometric-arithmetic indices are compared for chemical trees, starlike trees and general trees in Ref. [30], and the Wiener index and the Zagreb indices and the eccentric connectivity index for trees in Ref. [31]. In this note we prove that $E_{1}(T)>\xi^{c}(T)$ for chemical trees $T$, and for connected graph $G, \xi^{c}(G)>E_{2}(G)$ if $\Delta=n-1$ and $\xi^{c}(G)<E_{2}(G)$, otherwise. Moreover, we show that $G A_{2}>A B C_{G G}$ for paths and some class of bipartite graphs.

\section{PRELIMINARIES}

A connected graph with maximum vertex degree at most 4 is said to be a "molecular graph". ${ }^{[1]} \mathrm{A}$ tree in which the maximum vertex degree does not exceed 4 is said to be a "chemical tree". Denote, as usual, by $K_{1, n-1}, P_{n}, C_{n}$ and $K_{n}$ , the star, the path, the cycle and the complete graph on $n$ vertices, respectively. A double star of order $n$, denoted by $D S(p, q) \quad(p \geq q, n=p+q+2)$, is a tree, which is constructed by joining the central vertices of two stars $K_{1, p}$ and $K_{1, q}$. A vertex of a graph is said to be pendent if its neighborhood contains exactly one vertex. An edge of a graph is said to be pendent if one of its vertices is a pendent vertex. 


\section{COMPARISON BETWEEN $E_{1}$ AND $\xi^{C}$ OF GRAPHS}

In this section we compare the first Zagreb eccentricity index $\left(E_{1}\right)$ and the eccentric connectivity index $\left(\xi^{C}\right)$ for graphs. For $G \cong K_{1, n-1}, E_{1}(G)=4(n-1)+1>3(n-1)=\xi^{c}(G)$ and for $G \cong K_{n}(n>2), E_{1}(G)=n<n(n-1)=\xi^{c}(G)$. Therefore the first Zagreb eccentricity index and the eccentric connectivity index are incomparable on the class of general graphs. But we have the following theorem.

Theorem 3.1. Let $T$ be a chemical tree of order $n>2$. Then

$$
E_{1}(T)>\xi^{C}(T)
$$

Proof: Let $d$ be the diameter of tree $T$. Then $d \geq 2$. For $d=2, \quad T \cong K_{1, n-1}$ and hence $E_{1}(T)>\xi^{c}(T)$. For $d=3$, $T \cong D S(p, q) \quad(n=p+q+2)$. Then $\quad E_{1}(T)=9 n-10>$ $5 n-6=\xi^{c}(T)$. For $d=4$, the number of non-pendent vertices in $T$ is at most five and the number of pendent vertices in $T$ is at least two (since $T$ is a chemical tree). Exactly one non-pendent vertex, say $v_{i}$, has eccentricity 2 and all the other non-pendent vertices have eccentricity exactly 3 . For each non-pendent vertex $v_{j} \in V(T)(j \neq i)$,

$$
\varepsilon_{T}^{2}\left(v_{j}\right)-d_{T}\left(v_{j}\right) \cdot \varepsilon_{T}\left(v_{j}\right) \geq 9-12=-3 .
$$

For $v_{i} \in V(T)$,

$$
\varepsilon_{T}^{2}\left(v_{i}\right)-d_{T}\left(v_{i}\right) \cdot \varepsilon_{T}\left(v_{i}\right) \geq 4-8=-4 .
$$

For pendent vertex $v_{k} \in V(T)$ (vertex $v_{k}$ is on a diametral path),

$$
\varepsilon_{T}^{2}\left(v_{k}\right)-d_{T}\left(v_{k}\right) \cdot \varepsilon_{T}\left(v_{k}\right)=16-4=12 .
$$

All the other pendent vertices $v_{k} \in V(T)$, we have

$$
\varepsilon_{T}^{2}\left(v_{k}\right)-d_{T}\left(v_{k}\right) \cdot \varepsilon_{T}\left(v_{k}\right)>0 .
$$

Since the number of non-pendent vertices is at most five and the number of pendent vertices is at least two in $\mathrm{T}$, we have

$$
\sum_{i=1}^{n}\left(\varepsilon_{T}^{2}\left(v_{i}\right)-d_{T}\left(v_{i}\right) \cdot \varepsilon_{T}\left(v_{i}\right)\right)>0, \text { that is, } E_{1}(T)>\xi^{c}(T) .
$$

For $d=5$ or 6 , there are at most two vertices of eccentricity 3 with $\varepsilon_{T}^{2}\left(v_{i}\right)-d_{T}\left(v_{i}\right) \varepsilon_{T}\left(v_{i}\right) \geq-3$ and there are at least two pendent vertices of eccentricity 5 with $\varepsilon_{T}^{2}\left(v_{j}\right)-d_{T}\left(v_{j}\right) \varepsilon_{T}\left(v_{j}\right)=20$. For all other vertices $v_{k} \in V(G)$, the eccentricity is at least 4, therefore $\varepsilon_{T}^{2}\left(v_{k}\right)-d_{T}\left(v_{k}\right) \varepsilon_{T}\left(v_{k}\right) \geq 0$. Therefore again we get $E_{1}(T)>\xi^{c}(T)$.

For $d \geq 7$, we have $\varepsilon_{T}\left(v_{i}\right) \geq 4$ for any $v_{i} \in V(T)$. Since there is some vertex $v_{k}$ such that $\varepsilon_{T}\left(v_{k}\right)>4$ and $d_{T}\left(v_{i}\right) \leq 4$ for any $v_{i} \in V(T)$, again we get $\xi^{C}(T)<E_{1}(T)$. This completes the proof of the theorem.
In Ref. [29], we compared $M_{1}$ and $\xi^{c}$ for chemical tree and molecular graph. Moreover, we compare $E_{1}$ and $\xi^{c}$ for chemical tree in Theorem 3.1. We now obtain the following result for any connected graph.

Theorem 3.2. Let $G$ be a connected graph. Then

$$
M_{1}(G) \geq \xi^{c}(G) \text { or } E_{1}(G) \geq \xi^{c}(G) .
$$

Proof: We have

$$
\begin{aligned}
& M_{1}(G)+E_{1}(G)-2 \xi^{c}(G)=\sum_{i=1}^{n}\left(d_{G}^{2}\left(v_{i}\right)+\varepsilon_{G}^{2}\left(v_{i}\right)-\right. \\
& \left.2 d_{G}\left(v_{i}\right) \varepsilon_{G}\left(v_{i}\right)\right)=\sum_{i=1}^{n}\left(d_{G}\left(v_{i}\right)-\varepsilon_{G}\left(v_{i}\right)\right)^{2} \geq 0 .
\end{aligned}
$$

Thus

$$
M_{1}(G)+E_{1}(G) \geq 2 \xi^{c}(G)
$$

with equality holding if and only if $d_{G}\left(v_{i}\right)=\varepsilon_{G}\left(v_{i}\right)$, for every $i=1,2, \ldots, n$. Moreover, we have

$$
\left(M_{1}(G)-\xi^{c}(G)\right)+\left(E_{1}(G)-\xi^{c}(G)\right) \geq 0,
$$

which implies that $M_{1}(G) \geq \xi^{c}(G)$ or $E_{1}(G) \geq \xi^{c}(G)$. This completes the proof.

\section{COMPARISON BETWEEN $E_{2}$ AND $\xi^{C}$ OF GRAPHS}

We now compare $E_{2}(G)$ and $\xi^{c}(G)$ for any connected graph $G$.

Theorem 4.1. Let $G$ be a connected graph of order $n>1$ with maximum degree $\Delta$. If $\Delta=n-1$, then $E_{2}(G)<\xi^{C}(G)$. Otherwise, $E_{2}(G)>\xi^{C}(G)$.

Proof: First we assume that $\Delta=n-1$. Let $v_{1}, v_{2}, \ldots, v_{k}$ be the $k(\geq 1)$ vertices of degree $n-1$ in $G$.Then $\varepsilon_{G}\left(v_{i}\right)=1$ for $v_{i} \in V(G), i=1,2, \ldots, k$ and $\varepsilon_{G}\left(v_{i}\right)=2$ for $v_{i} \in V(G)$, $i=k+1, k+2, \ldots, n$. Now,

$$
\begin{aligned}
E_{2}(G)= & \sum_{v_{i} v_{j} \in(G)} \varepsilon_{G}\left(v_{i}\right) \cdot \varepsilon_{G}\left(v_{j}\right) \\
= & \sum_{\substack{v_{i} v_{j} \in E(G) \\
1 \leq i \neq j \leq k}} \varepsilon_{G}\left(v_{i}\right) \cdot \varepsilon_{G}\left(v_{j}\right) \\
& +\sum_{\substack{v_{i} v_{j} \in E(G) \\
1 \leq i \leq k, k+1 \leq j \leq n}} \varepsilon_{G}\left(v_{i}\right) \cdot \varepsilon_{G}\left(v_{j}\right)+\sum_{\substack{v_{i} v_{j} \in E(G) \\
k+1 \leq i \neq j \leq n}} \varepsilon_{G}\left(v_{i}\right) \cdot \varepsilon_{G}\left(v_{j}\right) \\
= & \frac{k(k-1)}{2}+k(n-k) 2+\left[m-\frac{k(k-1)}{2}-k(n-k)\right] 4 \\
= & 4 m-\frac{3 k(k-1)}{2}-2 k(n-k)
\end{aligned}
$$

and 


$$
\begin{aligned}
\xi^{c}(G) & =\sum_{i=1}^{n} d_{G}\left(v_{i}\right) \cdot \varepsilon_{G}\left(v_{i}\right) \\
& =\sum_{i=1}^{k} d_{G}\left(v_{i}\right) \cdot \varepsilon_{G}\left(v_{i}\right)+\sum_{i=k+1}^{n} d_{G}\left(v_{i}\right) \cdot \varepsilon_{G}\left(v_{i}\right) \\
& =k(n-1)+2 \sum_{i=k+1}^{n} d_{G}\left(v_{i}\right) \\
& =k(n-1)+2[2 m-k(n-1)]=4 m-k(n-1) .
\end{aligned}
$$

Since $n \geq 2$, one can easily see that

$$
4 m-k(n-1)>4 m-\frac{3 k(k-1)}{2}-2 k(n-k), \text { that is, } \xi^{c}(G)>E_{2}(G) \text {. }
$$

Next we assume that $\Delta \leq n-2$. Then $\varepsilon_{G}\left(v_{i}\right) \geq 2$ for every $v_{i} \in V(G)$. For any edge $v_{i} v_{j} \in E(G)$,

$$
\varepsilon_{G}\left(v_{i}\right)\left(\varepsilon_{G}\left(v_{j}\right)-1\right) \geq 2\left(\varepsilon_{G}\left(v_{j}\right)-1\right) \geq \varepsilon_{G}\left(v_{j}\right)
$$

that is,

$$
\varepsilon_{G}\left(v_{i}\right) \cdot \varepsilon_{G}\left(v_{j}\right) \geq \varepsilon_{G}\left(v_{i}\right)+\varepsilon_{G}\left(v_{j}\right) .
$$

Thus we have

$$
E_{2}(G)-\xi^{c}(G)=\sum_{v_{i} v_{j} \in E(G)}\left(\varepsilon_{G}\left(v_{i}\right) \cdot \varepsilon_{G}\left(v_{j}\right)-\varepsilon_{G}\left(v_{i}\right)-\varepsilon_{G}\left(v_{j}\right)\right) \geq 0 .
$$

This completes the proof of the theorem.

\section{COMPARISON BETWEEN $A B C_{G G}$ AND $G A_{2}$ OF GRAPHS}

Since each term of $G A_{2}$ and $A B C_{G G}$ are function of $n_{i}$ and $n_{j}$, it is interesting to compare of these two indices. We start with some examples:

\section{Example 1.}

$$
G A_{2}\left(K_{n}\right)=\frac{n(n-1)}{2}>0=A B C_{G G}\left(K_{n}\right) .
$$

Example 2.

$G A_{2}\left(C_{n}\right)=n$ and $A B C_{G G}\left(C_{n}\right)= \begin{cases}2 \sqrt{n-2} & \text { if } n \text { is even } \\ \frac{2 n}{n-1} \sqrt{n-3} & \text { if } n \text { is odd, }\end{cases}$ and therefore $G A_{2}\left(C_{n}\right)>A B C_{G G}\left(C_{n}\right)$.

Example 3. For $n \geq 5$,

$$
\begin{aligned}
G A_{2}\left(K_{1, n-1}\right) & =\frac{2(n-1) \sqrt{n-1}}{n}<\sqrt{(n-1)(n-2)} \\
& =A B C_{G G}\left(K_{1, n-1}\right) .
\end{aligned}
$$

From these examples, we can conclude that $G A_{2}$ index and $A B C_{G G}$ index are incomparable on the class of general graphs on $n$ vertices. So now we compare these two indices for special class of graphs. For path $P_{n}: v_{1} v_{2} \ldots v_{n-1} v_{n}$ $(n \geq 5)$, one can easily see that

$$
\frac{2 \sqrt{n_{i} n_{i+1}}}{n}>\sqrt{\frac{n-2}{n_{i} n_{i+1}}}, i=\left\lfloor\frac{n}{2}\right\rfloor, \text { and } \frac{2 \sqrt{n_{1} n_{2}}}{n}<\sqrt{\frac{n-2}{n_{1} n_{2}}} .
$$

Therefore there is a term in $G A_{2}\left(P_{n}\right)$ is greater than the corresponding term in $A B C_{G G}\left(P_{n}\right)$ and also there exists a term in $G A_{2}\left(P_{n}\right)$ is less than the corresponding term in $A B C_{G G}\left(P_{n}\right)$. So it is interesting to compare these two indices $\left(G A_{2}\right.$ and $\left.A B C_{G G}\right)$ for path $P_{n}$. For this we need the following result:

Lemma 5.1. For $1 \leq x \leq \frac{n-2}{4} \quad(n \geq 2)$ ，

$$
4 x^{3}-3 n x^{2}-\frac{n^{2}}{2} x+\frac{n^{3}}{4}>0 .
$$

Proof: Let us consider a function

$$
g(x)=4 x^{3}-3 n x^{2}-\frac{n^{2}}{2} x+\frac{n^{3}}{4}, 1 \leq x \leq \frac{n-2}{4} .
$$

Then $g^{\prime}(x)=12 x^{2}-6 n x-\frac{n^{2}}{2}$. Since $1 \leq x \leq \frac{n-2}{4}$ $(n \geq 2)$, one can easily see that $g^{\prime}(x)<0$ and hence $g^{4}(x)$ is a decreasing function on $1 \leq x \leq \frac{n-2}{4}$. Thus we have

$$
g(x) \geq g\left(\frac{n-2}{4}\right)=\frac{1}{8}\left(5 n^{2}-4\right)>0 .
$$

We are now ready to give the proof of $G A_{2}\left(P_{n}\right)>A B C_{G G}\left(P_{n}\right)$

Theorem 5.2. Let $n>2$ be a positive integer. Then $G A_{2}\left(P_{n}\right)>A B C_{G G}\left(P_{n}\right)$.

Proof: Let $n=2 p$ and $p=2 r+1$. We have

$$
\begin{aligned}
G A_{2}\left(P_{n}\right)= & \sum_{k=1}^{n-1} \frac{2 \sqrt{k(n-k)}}{n} \\
= & \frac{4}{n}[\sqrt{1 \cdot(n-1)}+\sqrt{2 \cdot(n-2)}+\cdots+\sqrt{(p-2) \cdot(p+2)} \\
& +\sqrt{(p-1) \cdot(p+1)}]+1 \\
= & \frac{4}{n} \sum_{x=1}^{r}\left[\sqrt{n x-x^{2}}+\sqrt{\frac{n^{2}}{4}-x^{2}}\right]+1 . \\
A B C_{2}\left(P_{n}\right)= & \sum_{k=1}^{n-1} \frac{\sqrt{n-2}}{\sqrt{k(n-k)}}+\frac{1}{\sqrt{2 \cdot(n-2)}}+\cdots \\
= & 2 \sqrt{n-2}\left[\frac{1}{\sqrt{1 \cdot(n-1)}}+\frac{1}{\sqrt{(p-2) \cdot(p+2)}}+\frac{2}{\sqrt{(p-1) \cdot(p+1)}}\right]+\frac{2}{n} \sqrt{n-2} \\
& \left.+\frac{1}{\sqrt{(p-1}}+\frac{1}{\sqrt{\frac{n^{2}}{4}-x^{2}}}\right]+\frac{2}{n} \sqrt{n-2} . \\
= & 2 \sqrt{n-2} \sum_{x=1}^{r}\left[\frac{1}{\sqrt{n x-x^{2}}}\right. \\
&
\end{aligned}
$$


Let us consider a function

$$
f(x)=\left(n x-x^{2}\right)\left(\frac{n^{2}}{4}-x^{2}\right), 1 \leq x \leq \frac{n-2}{4} .
$$

Then by Lemma 5.1, we have

$$
f^{\prime}(x)=4 x^{3}-3 n x^{2}-\frac{n^{2}}{2} x+\frac{n^{3}}{4}>0
$$

Therefore $f(x)$ is an increasing function on $1 \leq x \leq \frac{n-2}{4}$ and hence

$$
f(x) \geq f(1)=(n-1)\left(\frac{n^{2}}{4}-1\right) \geq \frac{n^{2}}{4}(n-2) .
$$

From the above, one can easily see that

$$
\sqrt{n x-x^{2}} \sqrt{\frac{n^{2}}{4}-x^{2}} \geq \frac{n \sqrt{n-2}}{2}, 1 \leq x \leq \frac{n-2}{4}
$$

that is,

$$
\begin{aligned}
\frac{4}{n}\left[\sqrt{n x-x^{2}}+\sqrt{\frac{n^{2}}{4}-x^{2}}\right] & \geq 2 \sqrt{n-2}\left[\frac{1}{\sqrt{n x-x^{2}}}+\frac{1}{\sqrt{\frac{n^{2}}{4}-x^{2}}}\right], \\
1 & \leq x \leq \frac{n-2}{4} .
\end{aligned}
$$

Since $n>2 \sqrt{n-2}$, from Eq. (5),Eq (6) and the above result, we have

$$
G A_{2}\left(P_{n}\right)>A B C_{2}\left(P_{n}\right)
$$

Similarly, one can easily prove the result for $n=2 p$, $p=2 r ; n=2 p+1, p=2 r+1 ; n=2 p+1, p=2 r$. This completes the proof of the theorem.

We now compare $G A_{2}$ and $A B C_{G G}$ for bipartite graph.

Theorem 5.3. Let $G$ be a bipartite graph of order $n$. If

$$
\left\lceil\frac{n}{2}-\sqrt{\frac{n^{2}}{4}-\frac{n}{2} \sqrt{n-2}}\right\rfloor \leq n_{i} \leq n_{j} \leq\left\lfloor\frac{n}{2}+\sqrt{\frac{n^{2}}{4}-\frac{n}{2} \sqrt{n-2}}\right\rfloor
$$

for any edge $v_{i} v_{j} \in E(G)$, then $G A_{2}(G) \geq A B C_{G G}(G)$.

Proof. Since $G$ is bipartite, $n_{i}+n_{j}=n$ for any edge $v_{i} v_{j} \in E(G)$. Therefore we have

$$
\begin{aligned}
G A_{2}(G)-A B C_{G G}(G) & =\sum_{v_{i} v_{j} \in E(G)}\left[\frac{2 \sqrt{n_{i} \cdot n_{j}}}{n_{i}+n_{j}}-\frac{\sqrt{n_{i}+n_{j}-2}}{\sqrt{n_{i} \cdot n_{j}}}\right] \\
& =\sum_{v_{i} v_{j} \in E(G)}\left[\frac{2 n_{i} n_{j}-n \sqrt{n-2}}{n \sqrt{n_{i} \cdot n_{j}}}\right] .
\end{aligned}
$$

The expression (7) is certainly non-negative if for every $v_{i} v_{j} \in E(G)$ we have

$$
2 n_{i} n_{j}-n \sqrt{n-2} \geq 0 \text {, that is, } 2 n_{i}\left(n-n_{i}\right)-n \sqrt{n-2} \geq 0,
$$

that is,

$$
n_{i}^{2}-n n_{i}+\frac{n}{2} \sqrt{n-2} \leq 0,
$$

that is,

$$
\frac{n}{2}-\sqrt{\frac{n^{2}}{4}-\frac{n}{2} \sqrt{n-2}} \leq n_{i} \leq n_{j} \leq \frac{n}{2}+\sqrt{\frac{n^{2}}{4}-\frac{n}{2} \sqrt{n-2}} .
$$

This completes the proof of the theorem.

Remark 5.4. We can construct several graphs such that the condition in Theorem 5.3 is satisfied. For $n=100$, we have

$$
\left\lceil\frac{n}{2}-\sqrt{\frac{n^{2}}{4}-\frac{n}{2} \sqrt{n-2}}\right\rceil=6,\left\lfloor\frac{n}{2}+\sqrt{\frac{n^{2}}{4}-\frac{n}{2} \sqrt{n-2}}\right\rfloor=94 .
$$

In the above theorem the condition is $6 \leq n_{i} \leq n_{j} \leq 94=$ $n-6$ for any edge $v_{i} v_{j} \in E(G)$. So we can find several graphs of order $n(=100)$ with that condition, for example, $C_{n}$. Moreover, we can construct several graphs such that $G A_{2}(G)>A B C_{G G}(G)$ without satisfy the condition in Theorem 5.3. For example, we have $G A_{2}\left(P_{n}\right)>A B C_{G G}\left(P_{n}\right)$, by Theorem 5.2.

\section{CONCLUSION}

Topological indices are graph invariants and are used for quantitative structure - activity relationship (QSAR) and quantitative structure - property relationship (QSPR) studies. Many topological indices have been defined in the literature and several of them have found applications as means to model physical, chemical, pharmaceutical and other properties of molecules. The eccentric connectivity index provides good correlations with regard to both physical and biological properties. In this note we presented that the eccentric connectivity index $\left(\xi^{c}\right)$ is less than the first Zagreb eccentricity index $\left(E_{1}\right)$ for chemical trees. For connected graph $G$, we prove that $\xi^{C}(G)>E_{2}(G)$ if $\Delta=n-1$ and $\xi^{C}(G)<E_{2}(G)$, otherwise. The GraovacGhorbani index is a distance-based analog of the atombond connectivity index, one of the most meaningful degree-based molecular structure descriptors. In this work, we show that the second geometric-arithmetic index $\left(G A_{2}\right)$ is greater than the Graovac-Ghorbani index $\left(A B C_{G G}\right)$ for paths and some class of bipartite graphs. There are many unsolved problems regarding the comparison between topological indices of graphs. The comparison between the first Zagreb eccentricity index $\left(E_{1}\right)$ and the eccentric connectivity index $\left(\xi^{c}\right)$, in the case of trees and general graphs is left as an open problem. The comparison between the second geometric-arithmetic index $\left(G A_{2}\right)$ and the GraovacGhorbani index $\left(A B C_{G G}\right)$ for general graphs remains a task for future. 
Acknowledgment. The author would like to thank Professor Nenad Trinajstić and all three anonymous referees for valuable comments which have considerably improved the presentation of this paper.

\section{REFERENCES}

[1] N. Trinajstić, Chemical Graph Theory, CRC Press, Boca Raton, 1983; $2^{\text {nd }}$ revised ed. 1992.

[2] R. Todeschini, V. Consonni, Handbook of Molecular Descriptors, Wiley-VCH, Wein-heim, 2000; R. Todeschini, V. Consonni, Molecular Descriptors for Chemoinformatics,Wiley-VCH, Weinheim, 2009, Vol. I, Vol. II.

[3] H. Wiener, J. Am. Chem. Soc. 1947, 69, 17.

[4] H. Hosoya, Bull. Chem. Soc. Japan 1971, 44, 2332.

[5] I. Gutman, Ber. Math.-Statist. Sekt. Forschungsz. Graz 1978, 103, 1.

[6] M. Randić, J. Am. Chem. Soc. 1975, 97, 6609.

[7] I. Gutman, N. Trinajstić, Chem. Phys. Lett. 1972, 17, 535.

[8] I. Gutman, K. C. Das, MATCH Commun. Math. Comput. Chem. 2004, 50, 83.

[9] S. Nikolić, I. M. Tolić, N. Trinajstić, I. Baućić, Croat. Chem. Acta 2000, 73, 909.

[10] D. Vukićević, N. Trinajstić, MATCH Commun. Math. Comput. Chem. 2005, 53, 111.

[11] D. Janežić, A. Milićević, S. Nikolić, N. Trinajstić, D. Vukićević, Croat. Chem. Acta 2007, 80, 541.

[12] M. Ghorbani, M. A. Hosseinzadeh, Filomat 2012, 26, 93.

[13] D. Vukićević, A. Graovac, Acta Chim. Slov. 2010, 57, 524.

[14] K. C. Das, D.-W. Lee, A. Graovac, Ars Mathematica Contemporanea 2013, 6, 117.
[15] R. Xing, B. Zhou, N. Trinajstić, Croat. Chem. Acta 2011, 84, 493.

[16] V. Sharma, R. Goswami, A. K. Madan, J. Chem. Inf. Comput. Sci. 1997, 37, 273.

[17] A. K. Madan, H. Dureja, Novel Molecular Structure Descriptors - Theory and Applications II (Eds.: I. Gutman, B. Furtula), University of Kragujevac, 2010, pp. 91-138.

[18] S. Gupta, M. Singh, A. K. Madan, J. Math. Anal. Appl. 2002, 266, 259.

[19] M. J. Morgan, S. Mukwembi, H. C. Swart, Discrete Math. 2011, 311, 1229.

[20] G. Fath-Tabar, B. Furtula, I. Gutman, J. Math. Chem. 2010, 47, 477.

[21] H. Hua, MATCH Commun. Math. Comput. Chem. 2010, 64, 631.

[22] B. Zhou, I. Gutman, B. Furtula, Z. Du, Chem. Phys. Lett. 2009, 482, 153.

[23] A. Graovac, M. Ghorbani, Acta Chim. Slov. 2010, 57, 609.

[24] W. Gao, W. Wang, J. Chem. 2014, 2014, 906254.

[25] K. C. Das, Appl. Math. Comput. 2016, 275, 353.

[26] K. C. Das, K. Xu, A. Graovac, Acta Chem. Slov. 2013, 60, 34.

[27] D. Dimitrov, B. Ikica, R. Škrekovski, Appl. Math. Comput. 2017, 293, 370.

[28] M. Rostami, M. S.-Haghighat, MATCH Commun. Math. Comput. Chem. 2014, 71, 21.

[29] K. C. Das, N. Trinajstić, Computers Math Appl. 2011, 62, 1758.

[30] K. C. Das, N. Trinajstić, Croat. Chem. Acta 2012, 85, 353.

[31] K. C. Das, H. Jeon, N. Trinajstić, Discrete Appl. Math. 2014, 171, 35. 